\title{
Atratividade e preferência para oviposição de Trialeurodes vaporariorum em genótipos de aboboreira
}

\author{
André C Alves ${ }^{1}$; André Luiz Lourenção ${ }^{1}$; Arlete M T de Melo ${ }^{1}$; Evandro da S Matos ${ }^{2}$ \\ ${ }^{1}$ IAC, C. Postal 28, 13012-970 Campinas-SP; ²Est. Exp. Rogers, Itatiba-SP; E-mail: andrecremasco@iac.sp.gov.br; andre@iac.sp.gov.br; \\ arlete@iac.sp.gov.br; evandro.matos@syngenta.com
}

\section{RESUMO}

O comportamento de 13 genótipos de aboboreira foi avaliado em relação à atratividade para adultos e à preferência para oviposição da mosca-branca Trialeurodes vaporariorum (West.) (Hemiptera: Aleyrodidae) em testes com e sem chance de escolha, em campo e em casa de vegetação. Os experimentos foram conduzidos com plantas de aboboreira em fase vegetativa, infestadas naturalmente no campo (atratividade para adultos e oviposição com chance de escolha) e artificialmente em casa de vegetação (oviposição sem chance de escolha). Dentre as aboboreiras tipo moita, o híbrido Arlika apresentou a menor atratividade para adultos e, entre os genótipos de crescimento rasteiro, não houve diferença em relação à atratividade. Quanto à preferência para oviposição em teste de livre escolha, 'Arlika', no grupo de moita, e 'Atlas', 'Sandy', 'Bárbara', 'Baianinha' e 'Caravela', no grupo das rasteiras, apresentaram os menores índices de oviposição, que se confirmaram no teste sem chance de escolha, indicando que a menor preferência para oviposição de $T$. vaporariorum nesses genótipos é estável.

Palavras-chave: Insecta, Aleyrodidae, mosca-branca, Cucurbita spp., resistência de plantas a insetos.

\begin{abstract}
Attractiveness and oviposition preference of Trialeurodes vaporariorum in squash genotypes

Thirteen squash genotypes were evaluated in relation to attractiveness and oviposition preference of Trialeurodes vaporariorum (West.) (Hemiptera: Aleyrodidae) under field and greenhouse conditions. In the field, two experiments were carried out, one being with bush squash and the other with summer squash genotypes. Plant infestation occurs naturally after seedling transplanting. By counting adults on the abaxial surfaces of the leaves, in the bush squash experiment, the hybrid 'Arlika' showed the lowest attractiveness; in the summer squash experiment, no difference was observed among genotypes. Regarding eggs counting, 'Arlika' (bush squash experiment), 'Atlas', 'Sandy', 'Barbara', 'Baianinha' and 'Caravela' (summer squash experiment) presented nonpreference for oviposition. Oviposition was also evaluated in two no-choice experiments, with artificial infestation, under greenhouse conditions. In both, the least preferred genotypes for oviposition in the freechoice test maintained the nonpreference, demonstrating that the resistance is stable.
\end{abstract}

Keywords: Insecta, Aleyrodidae, greenhouse whitefly, Cucurbita spp., host plant resistance

\section{(Recebido para publicação em 18 de abril de 2006; aceito em 18 de dezembro de 2006)}

$\mathrm{C}$ onhecida como mosca-branca de casa de vegetação, 'greenhouse whitefly' ou 'glasshouse whitefly', Trialeurodes vaporariorum (West.) (Hemiptera: Aleyrodidae) está distribuída pelo mundo todo, ocorrendo nos trópicos e subtrópicos em campo, e em regiões de clima temperado (Mound; Halsey, 1978; Kessing; Mau, 1991). T. vaporariorum se reproduz em ampla gama de plantas hospedeiras, sendo referidas espécies pertencentes a 82 famílias botânicas (Mound; Halsey, 1978). Dentre as plantas cultivadas, destacamse feijão, tomate, batata, abóbora, abobrinha, batata-doce, pepino, berinjela, pimentão, morango, melancia, poinsétia, rosa e outras ornamentais (Kessing; Mau, 1991).

Os danos ocasionados nem sempre são visíveis; todavia, grandes populações reduzem o vigor das plantas e a excreção de substância açucarada pelos insetos favorece o aparecimento de fumagina, que prejudica ainda mais a respiração e o desenvolvimento da planta (Kessing; Mau, 1991).

Em diversos municípios da região de Itatiba, SP, cultivos de tomate, feijão e abóboras têm sofrido severos ataques dessa mosca-branca, sendo a aplicação de defensivos agrícolas a alternativa mais adotada para seu controle. $\mathrm{O}$ uso excessivo desses produtos, além de induzir resistência do inseto aos inseticidas, tornando cada vez mais difícil seu controle, diminui a população de inimigos naturais e contamina o ambiente. Assim, o estudo de métodos alternativos de controle aumenta de importância em programas de manejo integrado. Dentre esses métodos, destaca-se a resistência varietal (Flint; Parks, 1990; Chu et al., 2001), método de controle que se integra de forma harmônica em programas de manejo integrado de pra- gas (Kogan, 1989).

O presente trabalho foi realizado com objetivo de determinar a atratividade para adultos e a preferência para oviposição de $T$. vaporariorum em treze genótipos de aboboreira (Cucurbita spp.), em testes com e sem chance de escolha.

\section{MATERIAL E MÉTODOS}

Os experimentos envolvendo atratividade e oviposição (livre chance de escolha) foram realizados em condição de campo e o de preferência para oviposição sem chance de escolha, em casa de vegetação, na Estação Experimental da Rogers, em Itatiba-SP.

Atratividade e preferência para oviposição em teste de livre escolha

Os genótipos avaliados constaram de aboboreiras de moita (Tabela 1) e de 
Tabela 1. Médias $( \pm \mathrm{EP})$ de atratividade para adultos (adultos $\left./ 10 \mathrm{~cm}^{2}\right)$ e preferência para oviposição (ovos $\left./ 10 \mathrm{~cm}^{2}\right)$ de $T$. vaporariorum, na face abaxial de folhas de cinco genótipos de aboboreira de moita (cv pepo). Itatiba-SP, Estação Experimental da Rogers, 2004/2005.

\begin{tabular}{|c|c|c|c|c|c|c|}
\hline \multirow[b]{2}{*}{ Genótipo } & \multirow[b]{2}{*}{ Procedência } & \multicolumn{3}{|c|}{ Número de adultos $/ 10 \mathrm{~cm} 2\left(^{*}\right)\left({ }^{* *}\right)$} & \multicolumn{2}{|c|}{ Ovos $/ 10 \mathrm{~cm} 2\left(^{*}\right)\left({ }^{* * *}\right)$} \\
\hline & & $\begin{array}{l}1^{\text {a }} \text { Avaliação } \\
(04 / 11 / 2004)\end{array}$ & $\begin{array}{l}2^{a} \text { Avaliação } \\
(19 / 11 / 2004)\end{array}$ & $\begin{array}{l}3^{a} \text { Avaliação } \\
(03 / 12 / 2004)\end{array}$ & $\begin{array}{l}\text { Livre escolha } \\
(03 / 12 / 2004)\end{array}$ & $\begin{array}{c}\text { Sem chance de } \\
\text { escolha } \\
(03 / 12 / 2005)\end{array}$ \\
\hline 'Golden Delight' & Rogers & $4,5 \pm 0,82 a$ & $18,1 \pm 6,39 a$ & $6,5 \pm 0,36 a$ & $71,6 \pm 6,48 a b$ & $18,3+0,84 a b$ \\
\hline 'Atlanta AG-303' & Horticeres & $3,5 \pm 1,04 a b$ & $12,2 \pm 3,87 a b$ & $5,6 \pm 0,75 a$ & $54,0 \pm 5,53 \quad b c$ & $13,6+0,60 a b$ \\
\hline 'Novita' & Sakata & $2,5 \pm 0,79 a b$ & $9,2 \pm 0,32 \mathrm{ab}$ & $2,2 \pm 0,13 a b$ & $42,7 \pm 4,61 \quad c$ & $12,5+2,12 a b$ \\
\hline 'Caserta' & Horticeres & $1,7 \pm 0,18 a b$ & $7,5 \pm 0,53 a b$ & $3,5 \pm 0,24 a b$ & $89,8 \pm 5,54 a$ & $19,3+3,43 a$ \\
\hline 'Arlika' & Rogers & $1,4 \pm 0,68 \quad b$ & $2,4 \pm 0,76 \quad b$ & $1,5 \pm 0,38 \quad b$ & $9,5 \pm 0,21 \quad d$ & $10,3+2,08 \quad b$ \\
\hline C.V\% & & 23,22 & 31,92 & 29,18 & 60,70 & 13,96 \\
\hline
\end{tabular}

*Médias seguidas pela mesma letra na coluna não diferem significativamente entre si pelo teste de Tukey (P $\leq 0,05)$; **Dados originais. Para análise estatística, os dados referentes ao número de adultos foram transformados em $\sqrt{x+0,5} ; * * *$ Dados originais. Para análise estatística, os dados referentes ao número de ovos foram transformados em $\sqrt{x}$.

crescimento rasteiro (Tabela 2). Devido a essa diferença no hábito de crescimento, foram conduzidos dois experimentos, montados com espaçamentos diferentes, visando atender às exigências de cada grupo. Os genótipos foram semeados em bandeja de poliestireno expandido de 72 células, preenchidas com substrato Plantimax ${ }^{\circledR}$. As mudas permaneceram por duas semanas em condições de cultivo protegido, até o transplante, que ocorreu em 14/09/2004. $\mathrm{O}$ espaçamento para aboboreiras de moita foi de $1 \mathrm{~m}$ entre covas, enquanto que, para as abóboras de crescimento rasteiro, foi de $4 \mathrm{~m} \times 3 \mathrm{~m}$. As covas possuíam $25 \mathrm{~cm}$ de diâmetro e a adubação foi de $200 \mathrm{~g}$ da fórmula 4-14-8. Efetuouse adubação de cobertura com $30 \mathrm{~g}$ de N-P-K 20-5-20 e a irrigação foi realizada diariamente, por aspersão.

Os dois experimentos foram delineados em blocos ao acaso. $\mathrm{O}$ experimento com genótipos tipo moita foi composto por de cinco tratamentos repetidos quatro vezes, totalizando 20 parcelas. O experimento com genótipos rasteiros constou de oito tratamentos repetidos quatro vezes, totalizando 32 parcelas. Cada parcela foi constituída de uma cova contendo duas plantas. A infestação de mosca-branca ocorreu de forma natural, com a migração de adultos presentes nas plantas invasoras e também nas culturas da estação experimental para as aboboreiras dos dois experimentos.

Com a finalidade de determinar, dentre os genótipos avaliados, os mais atrativos à mosca-branca, realizou-se con- tagem dos adultos presentes na face abaxial do terceiro par de folhas completamente desenvolvidas em três épocas, com intervalo de 15 dias. Após a realização da última contagem de adultos, foram coletadas duas folhas por parcela para avaliação da oviposição. A fim de preservar a qualidade desse material, as folhas foram acondicionadas em saco plástico e guardadas em geladeira, para posterior manuseio. Com auxílio de microscópico estereoscópico, sob aumento de 16x, foi realizada a contagem do número de ovos presentes na face abaxial das folhas. A seguir, efetuou-se a medição da área foliar em medidor LI-COR $3100 \mathrm{~A}$, para obtenção do número de ovos e de adultos por $10 \mathrm{~cm}^{2}$.

Preferência para oviposição em teste sem chance de escolha

Através de dois experimentos independentes, foi avaliada a preferência para oviposição da mosca-branca sem chance de escolha. No primeiro, avaliaram-se os genótipos tipo moita 'Golden Delight', 'Atlanta AG-303', 'Novita', 'Caserta' e 'Arlika'. No segundo experimento, avaliaram-se os genótipos tipo rasteiro 'Tetsukabuto', 'Bárbara', 'Baianinha', 'Caravela', 'Sandy' e 'Atlas', os quais, com exceção do híbrido 'Tetsukabuto', apresentaram os menores índices de oviposição no teste com chance de escolha. $\mathrm{O}$ delineamento experimental adotado para os dois experimentos foi o de blocos inteiramente casualizados, com quatro repetições. Cada parcela foi constituída de um vaso, com capacidade para $10 \mathrm{~L}$, contendo uma planta de aboboreira.
A infestação da mosca-branca foi realizada quando as plantas emitiram o terceiro par de folhas. Antes, em cada vaso, foi instalada uma armação de ferro $(35 \mathrm{~cm}$ de diâmetro superior $x 70 \mathrm{~cm}$ de altura), recoberta por tecido de 'voil'. Para infestação artificial, em cada vaso foram liberados cerca de 200 adultos de mosca-branca de idade desconhecida e mantidos por quatro dias. Esses adultos foram capturados em plantas de feijão e berinjela em uma propriedade do município de Itatiba, vizinho ao local dos experimentos.

A avaliação do número de ovos por $10 \mathrm{~cm}^{2}$ foi realizada de forma idêntica à dos experimentos realizados com livre escolha.

\section{Análise estatística}

Para todos os experimentos, o valor de número de ovos e de adultos de cada parcela foi obtido pela média das duas folhas selecionadas. Nos testes de livre escolha, as médias do número de adultos e do número de ovos foram transformadas em $\sqrt{x+0,5}$ e $\sqrt{x}$, respectivamente, e $\sqrt{x}$, nos testes sem chance de escolha, em . Foram realizadas análises de variância, sendo as médias comparadas pelo teste de Tukey $(\mathrm{P}<0,05)$, utilizando o programa estatístico Sanest.

\section{RESULTADOS E DISCUSSÃO}

Atratividade para adultos em testes com chance de escolha

A população de mosca-branca era alta e estava distribuída em toda a Esta- 
Tabela 2. Médias ( \pm EP) de atratividade para adultos $\left(\right.$ adultos $/ 10 \mathrm{~cm}^{2}$ ) e preferência para oviposição (ovos/10 $\mathrm{cm}^{2}$ ) de $T$. vaporariorum, na face abaxial de folhas de oito genótipos de aboboreira de crescimento rasteiro. Itatiba-SP, Estação Experimental da Rogers, 2004/2005.

\begin{tabular}{|c|c|c|c|c|c|c|c|}
\hline \multirow[b]{2}{*}{ Genótipo } & \multirow[b]{2}{*}{ Procedência } & \multirow[b]{2}{*}{ Espécie } & \multicolumn{3}{|c|}{ Número de adultos $/ 10 \mathrm{~cm} 2\left({ }^{*}\right)\left({ }^{* *}\right)$} & \multicolumn{2}{|c|}{ Ovos/10 cm2 $\left({ }^{*}\right)\left({ }^{* *}\right)$} \\
\hline & & & $\begin{array}{l}1^{\text {a }} \text { Avaliação } \\
\text { (04/11/2004) }\end{array}$ & $\begin{array}{l}2^{a} \text { Avaliação } \\
\text { (19/11/2004) }\end{array}$ & $\begin{array}{l}3^{\text {a }} \text { Avaliação } \\
\text { (03/12/2004) }\end{array}$ & $\begin{array}{l}\text { Livre escolha } \\
(03 / 12 / 2004)\end{array}$ & $\begin{array}{c}\text { Sem chance } \\
\text { de escolha } \\
(03 / 12 / 2005)\end{array}$ \\
\hline 'Menina Brasileira' & $\mathrm{IAC}$ & C. moschata & $1,0 \pm 0,45 a$ & $1,9 \pm 0,27 a$ & $3,1 \pm 1,08 \mathrm{a}$ & $13,8 \pm 3,50 a b$ & \\
\hline 'Barbara' & Sakata & C. moschata & $0,7 \pm 0,22 \mathrm{a}$ & $1,7 \pm 0,33 a$ & $2,7 \pm 0,61 \mathrm{a}$ & $7,8 \pm 1,62 \quad b c$ & $12,9 \pm 2,63 b$ \\
\hline 'Caravela' & $\mathrm{IAC}$ & C. moschata & $0,6 \pm 0,16 \mathrm{a}$ & $1,1 \pm 0,35 a$ & $2,6 \pm 0,78 a$ & $5,7 \pm 0,45$ bc & $5,9 \pm 0,93 b$ \\
\hline 'Exposição' & Sakata & C. moschata & $0,4 \pm 0,12 \mathrm{a}$ & $0,9 \pm 0,31 \mathrm{a}$ & $4,4 \pm 1,22 \mathrm{a}$ & $13,4 \pm 2,36 a b$ & \\
\hline 'Baianinha' & $\mathrm{IAC}$ & C. maxima & $0,3 \pm 0,10 \mathrm{a}$ & $0,9 \pm 0,16 a$ & $2,3 \pm 0,96 \mathrm{a}$ & $4,8 \pm 1,70 \quad b c$ & $7,5 \pm 1,34 \quad b$ \\
\hline 'Sandy' & Sakata & C. moschata & $0,3 \pm 0,08$ a & $0,9 \pm 0,31 \mathrm{a}$ & $3,6 \pm 1,72 \mathrm{a}$ & $3,8 \pm 1,66 \quad c$ & $4,0 \pm 0,58 \quad b$ \\
\hline 'Atlas' & Sakata & C. moschata & $0,3 \pm 0,07 a$ & $1,0 \pm 0,22 a$ & $1,8 \pm 0,22 \mathrm{a}$ & $6,1 \pm 0,86 \quad b c$ & $3,2 \pm 0,24 \quad b$ \\
\hline 'Tetsukabuto' & Sakata & $\begin{array}{l}\text { C. maxima } \mathrm{x} \\
\text { C. moschata }\end{array}$ & $0,3 \pm 0,06 \mathrm{a}$ & $1,0 \pm 0,25 a$ & $8,6 \pm 3,48 \mathrm{a}$ & $22,9 \pm 3,46 a$ & $42,2 \pm 11,47 a$ \\
\hline C.V\% & & & 17,74 & 20,18 & 36,45 & 22,90 & 28,77 \\
\hline
\end{tabular}

*Médias seguidas pela mesma letra na coluna não diferem significativamente entre si pelo teste de Tukey (P $\leq 0,05)$; **Dados originais. Para análise estatística, os dados referentes ao número de adultos foram transformados em $\sqrt{x+0,5} ; * * *$ Dados originais. Para análise estatística, os dados referentes ao número de ovos foram transformados em $\sqrt{x}$.

ção Experimental e em diversos cultivos da região. Em campo, foram observadas colônias do inseto em tomate, couve, aboboreira e também em plantas invasoras, como serralha (Sonchus oleraceus) e picão-preto (Bidens pilosa); em cultivo protegido, plantas ornamentais como gérbera, prímula, crisântemo, entre outras, apresentavam-se colonizadas por esta praga (Lourenção et al., 2006). Dessa forma, logo após o transplante para campo, houve infestação natural nos experimentos.

Para abóbora de moita, na primeira avaliação realizada, verificou-se que 'Arlika', com 1,4 adultos/10 cm², apresentou a menor presença de adultos da mosca-branca, diferindo de 'Golden Delight', a de maior atratividade, com 4,5 adultos $/ 10 \mathrm{~cm}^{2}$; os demais genótipos apresentaram comportamento intermediário (Tabela 1). O mesmo foi observado na segunda e terceira avaliações, quando 'Arlika' (2,4 e 1,5 adultos/ 10 $\mathrm{cm}^{2}$ respectivamente) foi o genótipo menos atrativo e novamente diferiu de 'Golden Delight', que apresentou 18,1 e 6,5 adultos $/ 10 \mathrm{~cm}^{2}$. Em estudo de Alves et al. (2005), em que se avaliou a resistência de aboboreiras a outra espécie de mosca-branca, Bemisia tabaci biótipo B, verificou-se que, dentre os genótipos tipo moita, 'Golden Delight' também foi o mais atrativo aos adultos.

No experimento envolvendo aboboreiras de crescimento rasteiro, as médias de adultos $/ 10 \mathrm{~cm}^{2}$ (Tabela 2) foram comparativamente mais baixas que as médias do experimento de aboboreira de moita (Tabela 1), o que sugere maior atratividade de genótipos deste grupo para essa mosca-branca, uma vez que os dois experimentos foram instalados de forma adjacente, na mesma época e com os mesmos tratos culturais. Na primeira avaliação, as médias oscilaram de 0,3 a 1,0 adultos/ $10 \mathrm{~cm}^{2}$; na segunda, variaram de 0,9 a 1,9 adultos $/ 10 \mathrm{~cm}^{2} \mathrm{e}$, na terceira, verificou-se a maior infestação: 1,8 a 8,6 adultos $/ 10 \mathrm{~cm}^{2}$. Nas três avaliações não foram detectadas diferenças entre os genótipos. Esses resultados indicam que possivelmente não haja níveis significativos de fatores de atração e/ou de repelência nas plantas dos diferentes genótipos que possam afetar a atratividade e a permanência dessa espécie de mosca-branca. Para B. tabaci biótipo $\mathrm{B}$, houve discriminação quanto à presença de adultos em aboboreiras do tipo rasteiro, em experimento envolvendo as mesmas cultivares, com exceção de 'Sandy' (Alves et al., 2005). É possível, também, que os níveis de infestação tenham sido insuficientes para discriminar a resistência entre os genótipos avaliados.

Preferência para oviposição em testes com chance de escolha

Dentre as aboboreiras tipo moita, 'Arlika' apresentou o menor número de ovos de mosca-branca, diferindo de todos os outros genótipos (Tabela 1). Bai- xa oviposição por B. tabaci biótipo B nesse híbrido também foi observada em teste com chance de escolha por Alves et al. (2005). Esse híbrido também foi o menos atrativo aos adultos de $T$. vaporariorum, indicando associação entre adultos e oviposição. Para B. tabaci biótipo B em soja, foi detectada correlação positiva e significativa entre adultos e oviposição (Valle; Lourenção, 2002), o que pode estar ocorrendo também para T. vaporariorum em aboboreiras. Entre os genótipos mais ovipositados, destacaram-se 'Caserta' e 'Golden Delight'; em posição intermediária, mas também apresentando elevado número de ovos em relação a 'Arlika', situaram-se 'Atlanta AG-303' e 'Novita' (Tabela 1).

Com relação às abóboras de crescimento rasteiro, o híbrido Sandy foi o genótipo menos ovipositado, diferindo de 'Tetsukabuto', 'Menina Brasileira' e 'Exposição' (Tabela 2); já os genótipos 'Baianinha' e 'Bárbara' não diferiram de 'Sandy'. Da mesma forma ao observado na avaliação de atratividade, houve maior número de adultos de moscabranca em genótipos tipo moita em relação a aboboreiras de porte rasteiro, verificando-se também tendência de maior oviposição em genótipos tipo moita (Tabelas 1, 2). Para B. tabaci biótipo B, também ‘Tetsukabuto' destacou-se como o genótipo mais ovipositado (Alves et al., 2005).

Em teste com chance de escolha para B. tabaci biótipo B, Baldin et al. (2000) 
avaliaram a preferência para oviposição em dez acessos não comerciais de aboboreiras de crescimento rasteiro, usando 'Exposição' como testemunha. Embora com comportamento intermediário, essa cultivar sofreu elevada oviposição, a exemplo do ocorrido com T. vaporariorum.

Preferência para oviposição em testes sem chance de escolha

Para as aboboreiras tipo moita, 'Arlika' diferiu de 'Caserta' e apresentou o menor número de ovos de $T$. vaporariorum, porém sem diferir dos demais genótipos (Tabela 1). Para $B$. tabaci biótipo B, 'Caserta' situou-se entre as aboboreiras menos ovipositadas em testes com e sem chance de escolha (Alves et al., 2005), o que sugere a presença de mecanismos distintos de resistência a essas duas espécies de moscabranca em aboboreiras.

Em relação às abóboras de hábito de crescimento rasteiro, os híbridos Atlas, Sandy, Bárbara e as cultivares Caravela e Baianinha foram menos ovipositados que 'Tetsukabuto', que se mostrou suscetível (Tabela 2). Da mesma forma que para as abóboras tipo moita, o teste sem chance de escolha para as abóboras de crescimento rasteiro confirmou a menor oviposição de $T$. vaporariorum nesses cinco genótipos, indicando que a nãopreferência observada em condições de livre escolha é estável, ou seja, a menor oviposição se mantém mesmo quando o inseto não tem outros genótipos mais adequados.

Considerando-se todas as avaliações, verifica-se que, dentre o germoplasma tipo moita, 'Arlika' exibe baixa atratividade aos adultos de $T$. vaporariorum e que, na ausência de chance de escolha, esse híbrido é pouco ovipositado, características vantajosas para plantio em regiões de ocorrência dessa praga. Por sua vez, 'Caserta', pouco ovipositada por B. tabaci biótipo B (Alves et al., 2005), e 'Golden Delight', tiveram altos níveis de oviposição no presente estudo, caracterizando-se como suscetíveis a $T$. vaporariorum. Entre as aboboreiras rasteiras, 'Atlas', 'Sandy', 'Caravela' e 'Baianinha' tiveram baixo nível de oviposição, enquanto 'Tetsukabuto' foi a mais ovipositada, demonstrando suscetibilidade a essa mosca-branca.

\section{REFERÊNCIAS}

ALVES AC; LOURENÇÃO AL; MELO AMT. 2005. Resistência de genótipos de aboboreira a Bemisia tabaci biótipo B (Hemiptera: Aleyrodidae). Neotropical Entomology 34: 973-979.

BALDIN ELL; TOSCANO LC; LIMA ACS; LARA FM; BOIÇA JUNIOR AL. 2000. Preferencia para oviposición de Bemisia tabaci biótipo "B" por genótipos de Cucurbita moschata y Cucurbita máxima. Boletin de Sanidad Vegetal 26: 409-413.
CHU CC; FREEMAN TP; BUCKNER JS; HENNEBERRY TJ; NELSON DR; NATWICK ET. 2001. Suscetibility of upland cotton cultivars to Bemisia tabaci biotype B (Homoptera: Aleyrodidae) in relation to leaf age and trichome density. Annals of Entomological Society of America 94: 743749.

FLINT HM; PARKS NJ. 1990. Infestation of germoplasm lines and cultivars of cotton in Arizona by whitefly nymphs (Homoptera: Aleyrodidae). Journal of Entomological Science 25: 223-229.

KESSING JLM; MAU RFL. 2004. Trialeurodes vaporariorum (Westwood). Crop Knowledge Master, 1991 (Disponível na internet). Acessado em 16 dezembro 2004.

KOGAN M. 1989. Plant resistance in soybean insect control. In: CONFERENCIA MUNDIAL DE INVESTIGACION EN SOJA, 4. Actas. Buenos Aires: Orientación Gráfica Editora SRL 3: 1519-1525.

LOURENÇÃO AL; ALVES AC; FUGI CGQ; MATOS ER. Surtos populacionais de Trialeurodes vaporariorum (West.) (Hemiptera: Aleyrodidae) em campo no estado de São Paulo. In: CONGRESSO BRASILEIRO DE ENTOMOLOGIA, 21. Resumos...Recife: SEB (CD-ROM).

MOUND LA; HALSEY SH. 1978. Whitefly of the World. A systematic catalogue of the Aleyrodidae (Homoptera) with host plant and natural enemy data. London, British Museum (Natural History). John Wiley and Sons. New York. 340p.

VALLE GE; LOURENÇÃO AL. 2002. Resistência de genótipos de soja a Bemisia tabaci (Genn.) biótipo B (Hemiptera: Aleyrodidae). Neotropical Entomology 31: 285-295. 